\title{
Adaptive Switching Median Filter for Impulse Noise Removal Based on Support Vector Machines
}

\author{
Dae Geun Lee ${ }^{a}$, Min Jae Park ${ }^{b}$, Jeong Ok Kim ${ }^{b}$, Do Yoon $\mathrm{Kim}^{b}$, Dong Wook Kim ${ }^{c}$, \\ Dong Hoon Lim ${ }^{1, d}$ \\ ${ }^{a}$ Computer Science and Engineering, Seoul National University \\ ${ }^{b}$ Korea Advanced Institute of Science and Technology \\ ${ }^{c}$ Department of Statistics, Busan National University \\ ${ }^{d}$ Department of Information Statistics and RINS, Gyeongsang National University
}

\begin{abstract}
This paper proposes a powerful SVM-ASM filter, the adaptive switching median(ASM) filter based on support vector machines(SVMs), to effectively reduce impulse noise in corrupted images while preserving image details and features. The proposed SVM-ASM filter is composed of two stages: SVM impulse detection and ASM filtering. SVM impulse detection determines whether the pixels are corrupted by noise or not according to an optimal discrimination function. ASM filtering implements the image filtering with a variable window size to effectively remove the noisy pixels determined by the SVM impulse detection. Experimental results show that the SVM-ASM filter performs significantly better than many other existing filters for denoising impulse noise even in highly corrupted images with regard to noise suppression and detail preservation. The SVM-ASM filter is also extremely robust with respect to various test images and various percentages of image noise.
\end{abstract}

Keywords: Support vector machine, adaptive switching median filter, SVM-ASM filter, impulse noise, impulse detection.

\section{Introduction}

Impulse noise is often caused by malfunctioning pixels in camera sensors, faulty hardware memory locations, or image transmission in a noisy communication channel. Impulse noise can severely degrade image quality and cause significant information loss; therefore, it it is necessary to eliminate noise before subsequent processing such as edge detection, image segmentation and object recognition (Lim and Jang, 2002; Lim, 2006).

The objective of noise removal is to suppress noise while preserving the integrity of the edges and the detail information in the original images. Many techniques have been proposed to remove impulse noise.

One of the most popular techniques is the median (MED) filter (Gonzalez and Woods, 1992) that can suppress noise and has a high computational efficiency. However, the MED filter tends to blur fine details or destroy edges when it is uniformly applied to an image because it is space-invariant and replaces every pixel with the median value of the filter window regardless of whether the pixel is noise-free of noise-corrupted. A simple extension to the MED filter incorporates spatial information

\footnotetext{
This work was supported by the National Research Foundation of Korea(NRF) grant funded by the Korea government(No. 2011-0010089).

${ }^{1}$ Corresponding author: Professor, Department of Information Statistics and RINS, Gyeongsang National University, Jinju 660-701, Korea. E-mail: dhlim@gsnu.ac.kr
} 


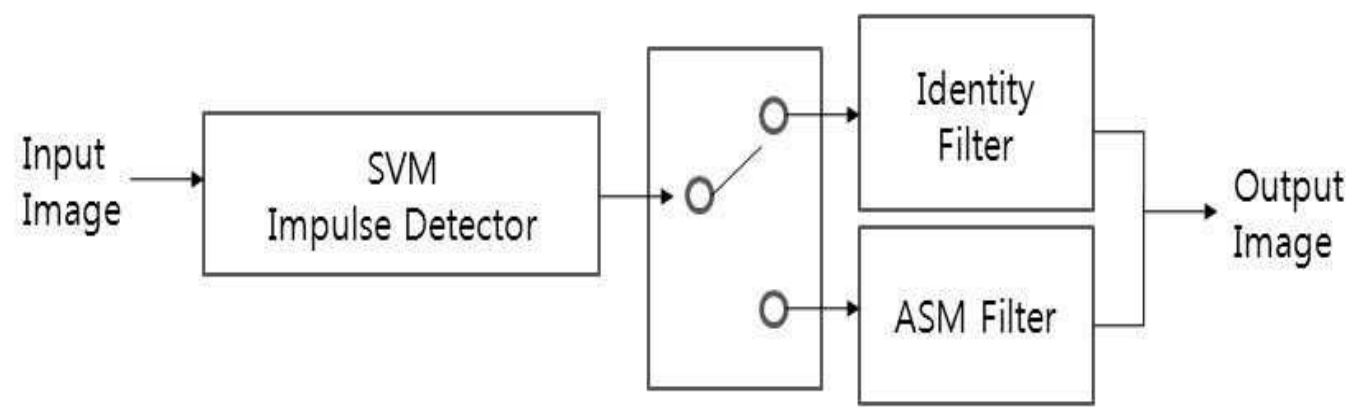

Figure 1: Block diagram of SVM-ASM filter

into the filtering process is the center-weighted median(CWM) filter (Ko and Lee, 1991) that gives more weight to the central value in the window. This leads to an improvement in detail preservation at the expense of lower noise suppression.

Conventional median filters are spatially invariant operators incapable of making any distinction between the corrupted and uncorrupted pixels of the noisy image. Hence, these filters distort the uncorrupted regions of the image during the restoration of the corrupted regions and cause the useful information in the image to be lost. The most straightforward solution to this problem is the switching median filter(SWM-I and SWM-II) (Sun and Neuvo, 1994), which is obtained combining the median filter with an impulse detector. In this approach, the impulse detector aims to determine whether the center pixel of a given filtering window is corrupted or not. If the center pixel is identified by the detector as a corrupted pixel, it is then replaced with the output of the median filter (otherwise it is left unchanged). Signal-dependent rank-ordered mean(SD-ROM) filter (Abreu and Mitra, 1995) is another switching filter utilizing rank-order information for impulse noise removal. The structure of the filter is the same as a switching median filter except that the median filter is replaced with a rankordered mean filter. These switching median filters yield satisfactory results when the parameters concerned are properly set; however, it is not easy to exploit fixed decision-making parameters, since the parameters are obtained at a pre-assumed noise density level.

In Apalkov et al. (2005) and Zvonarev et al. (2005a, 2005b), two-stage noise removal algorithms based on neural network and an adaptive switching median(NN-ASM) filter were proposed; however, they work only on fixed impulse noise and they are computationally expensive.

Lin and Yu (2004), and Liu et al. (2006) have proposed two-stage filters, respectively denoted by SVM-NFR filter and SVM-EPR filter, based on support vector machines(SVMs) for removing impulse noise, with a scheme similar to (Apalkov et al., 2005; Zvonarev et al., 2005a, 2005b). In the first phase, they use SVMs to identify pixels which are likely to be noise. In the second phase, these noise candidates are restored by the noise-free reduction median(NFR) filter or the edge-preserving regularization(EPR) (Chan et al., 2005), respectively. These filters mainly focus on the first phase, but they have shortcomings that mistakenly leave any noisy pixels undetected or mis-detect uncorrupted pixels, especially when the images are highly corrupted.

This paper proposes a powerful SVM-ASM filter, the adaptive switching median(ASM) filter based on SVMs, for effectively reducing impulse noise in corrupted images while preserving image details and features. The proposed SVM-ASM filter is mainly composed of two components: SVM impulse detector and ASM filter (Figure 1). The proposed SVM detector is generated by adding a new variable based on the rank order arrangement of the pixels in the sliding window to improve the capability of impulse noise detection. The former determines whether the pixels are corrupted by 
noise or not according to an optimal discrimination function. The latter implements the image filtering with variable window size on the noisy pixels to effectively remove the noisy pixels determined by the former. Due to its unbiased detection-filtering criteria, this filter treats both fixed-valued and random-valued noise even in highly corrupted images.

The remainder of the paper is organized as follows. Section 2 briefly reviews SVMs. Section 3 presents the details of our SVM-ASM filter. Section 4 provides experimental results to demonstrate the performance of the SVM-ASM filter, and Section 5 presents conclusions.

\section{Review of SVMs}

SVMs are a set of related supervised learning methods based on margin maximization introduced by Vapnik et al. (1998). SVMs perform binary pattern classification by finding an optimal discrimination function that is induced from training examples. Let $\left\{\left(x_{i}, y_{i}\right), i=1, \ldots, N\right\}$ be a set of training examples, each example $x_{i} \in R^{d}, d$ being the dimension of the input space, belongs to a class labeled by $y_{i} \in\{-1,1\}$ with a hyperplane $w x+b=0$, which divides the set of examples such that all the points with the same label are on the same side of the hyperplane. The optimal separating hyperplane is obtained just the set of examples is separated without error and the margin is maximal.

We now formulate the primal problem of the SVM:

$$
\begin{array}{ll}
\operatorname{minimize} & \frac{1}{2}\|w\|^{2}, \\
\text { subject to } & y_{i}\left[\left(w \cdot x_{i}\right)+b\right] \geq 1, \quad i=1, \ldots, N .
\end{array}
$$

We maximize the margin, subject to the constraints that all training cases fall on either side of the support hyper-planes. The data-cases that lie on the hyperplane are called support vectors, since they support the hyper-planes and determine the solution to the problem.

The primal problem can be solved by a quadratic program. Hence we transform to the dual formulation by first writing the problem using a Lagrangian,

$$
L(w, b, \alpha)=\frac{1}{2}\|w\|^{2}-\sum_{i=1}^{N} \alpha_{i}\left[y_{i}\left[\left(w \cdot x_{i}\right)+b\right]-1\right] .
$$

This is done by taking derivatives with respect to $w$ and $b$ and solving,

$$
\begin{array}{r}
w-\sum_{i=0}^{N} \alpha_{i} y_{i} x_{i}=0, \\
\sum_{i=0}^{N} \alpha_{i} y_{i}=0 .
\end{array}
$$

Inserting this back into the Lagrangian we obtain what is known the dual problem,

$$
\begin{aligned}
& \operatorname{maximize} L_{D}=\sum_{i=1}^{N} \alpha_{i}-\frac{1}{2} \sum_{i, j=1}^{N} \alpha_{i} \alpha_{j} y_{i} y_{j} x_{i} x_{j}, \\
& \text { subject to } \sum_{i=0}^{N} \alpha_{i} y_{i}=0, \quad \alpha_{i} \geq 0, i=1, \ldots, N
\end{aligned}
$$


In this work, we need to consider the linearly nonseparable case and the nonlinear case because the training noisy examples have both. The nonlinear discrimination function is of the form

$$
f(x)=\operatorname{sign}\left(\sum_{i=1}^{N} \alpha_{i} y_{i} K\left(x_{i}, x\right)+b\right),
$$

where $K\left(x_{i}, x\right)$ is a positive definite kernel function. A few kernel functions have been found to work well in a wide variety of applications, i.e.:

$$
\begin{aligned}
& \text { polynomial kernel : } K(x, y)=\left(\gamma x^{T} y+r\right)^{p}, \quad \gamma>0, \\
& \text { radial basis function(RBF) kernel : } K(x, y)=\exp \left(-\gamma\|x-y\|^{2}\right), \quad \gamma>0, \\
& \text { sigmoid kernel : } K(x, y)=\tanh \left(\gamma x^{T} y+r\right),
\end{aligned}
$$

where $\gamma, r$, and $p$ are kernel parameters.

The kernel used in our work is the RBF since it offers the best results with a reduced number of support vectors (Cheng et al., 2005; Ganapathiraju et al., 2004; Moreno et al., 2001; Moreno et al., 2003). This kernel nonlinearly maps samples into a higher dimensional space so it (unlike the linear kernel) can handle the case when the relation between class labels and attributes is nonlinear. Keerthi and Lin (2003) show that the linear kernel is a special case of the RBF. The sigmoid kernel behaves like RBF for certain parameters (Lin and Lin, 2003). The RBF function has fewer parameters to tune than a polynomial kernel, and the RBF kernel has less numerical difficulties.

When some data into the sets cannot be separated, the SVMs can introduce slack variables $(\xi \geq 0)$ to relax the minimization constraints. Then, the optimization problem takes the form,

$$
\begin{aligned}
& \operatorname{minimize} \frac{1}{2}\|w\|^{2}+C \sum_{i=0}^{N} \xi_{i}, \\
& \text { subject to } y_{i}\left[\left(w \cdot x_{i}\right)+b\right] \geq 1-\xi_{i}, \quad i=1, \ldots, N .
\end{aligned}
$$

A penalty term $C$ that makes more or less important the misclassification error in the minimization process is included.

\section{The SVM-ASM Filter}

Before introducing the proposed SVM-ASM filter, some notations must be defined. Let $K=\left\{\left(k_{1}, k_{2}\right) \mid\right.$ $\left.1 \leq k_{1} \leq H, 1 \leq k_{2} \leq W\right\}$ denote the pixel coordinates of the noisy image corrupted by impulse noise, where $H$ and $W$ are the image height and width, respectively. Let $x(k)$ represent the input pixel value of the noisy image at location $k \in K$. At each location $k$, the observed filter window $w\{k\}$ whose size is $N=2 n+1$ ( $n$ is a non-negative integer) is defined in terms of the coordinates symmetrically surrounding the input pixel $x(k)$.

$$
w\{k\}=\left\{x_{i}(k): i=1,2, \ldots, n, n+1, \ldots, N\right\},
$$

where the input pixel $x(k)=x_{n+1}(k)$ is the center pixel. For example, we consider a $3 \times 3$ filter window centered around $x(k)=x_{5}(k)$ such that

$$
w\{k\}=\left\{x_{1}(k), \ldots, x_{4}(k), x_{5}(k), x_{6}(k), \ldots x_{9}(k)\right\} .
$$




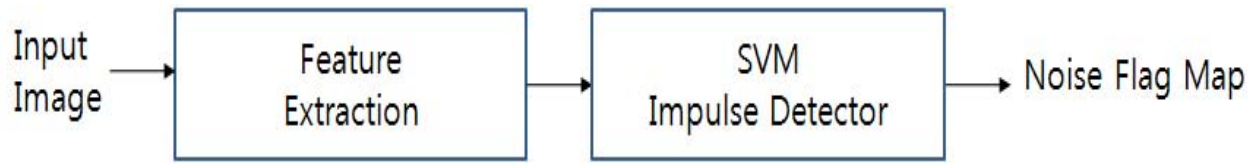

Figure 2: The impulse detection process of our proposed filter

The output value from a MED filter is denoted as $\operatorname{med}(w\{k\})$ at location $k$ in a filter window of size $N$ as follows:

$$
\operatorname{med}(w\{k\})=\operatorname{med}\left(x_{1}(k), \ldots, x_{n}(k), x_{n+1}(k), \ldots, x_{N}(k)\right),
$$

where med denotes the median operation. The output of the CWM filter is also denoted as $\operatorname{med}\left(w_{c}\{k\}\right)$ at location $k$ as follows:

$$
\operatorname{med}\left(w_{c}\{k\}\right)=\operatorname{med}\left(x_{1}(k), \ldots, x_{n}(k), c \diamond x_{n+1}(k), \ldots, x_{N}(k)\right),
$$

where $c(c=1,3,5, \ldots, N)$ denotes the center weight and $\diamond$ represents the repetition operation.

\subsection{SVM for impulse detection}

The input pixels are first identified by the SVM impulse detector to be either noise-free or noisecorrupted. A binary noise map denoted as $F$ is used to indicate whether the pixels have been detected as impulses in the test image,

$$
F=\{f(x) \mid k \in K\} .
$$

If $f(k)$ equals 1 , it implies the pixel $x(k)$ is corrupted by noise; otherwise, $x(k)$ is noise-free.

The SVM impulse detector uses the local characteristics in the filter window as observation vectors to classify the signals into two classes: noise-free and noise-corrupted signals. This design of the detection approach consists of two steps: feature extraction and training the SVM impulse detector, as shown in Figure 2.

\subsubsection{Feature extraction}

In this work, the following four feature variables $u(k), v(k), q(k)$ and $r(k)$ can be defined to generate the observation vector $O(k)$ as the input data of the SVM impulse detector. The amplitudes of most impulse noise exceed those of the fine changes of the signals. Thus, the difference between the input pixel $x(k)$ and the median of the filter window $w(k)$ provides efficient evidence that can be used to identify a noisy pixel.

\section{Definition 1.}

$$
u(k)=|x(k)-\operatorname{med}(w\{k\})| .
$$

The variable $u(k)$ is a measure used in providing a simple measurement to detect impulse. A large value of $u(k)$ indicates that the input $x(k)$ is different to the median value of the filter window $w\{k\}$. The central pixel $x(k)$ is corrupted by impulse noise. Most impulse noises can be detected using the variable $u(k)$ as the indicator. Nevertheless, using only the variable $u(k)$ to detect the noise raises the following three problems.

The first problem is that if only the variable $u(k)$ is considered to determine whether an impulse noise exists, then fully separating the impulse noise is difficult. For instance, an image usually has 


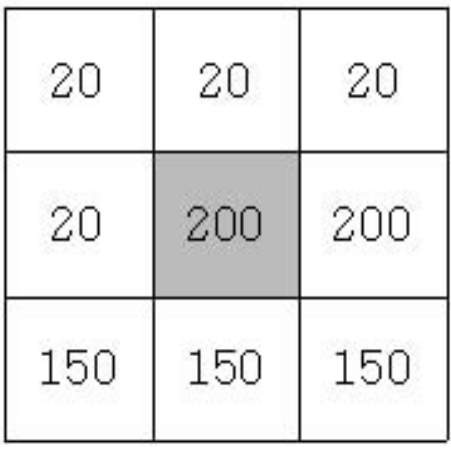

Figure 3: The pixel $x(k)$ with value 200 as noise

line components with a width of just one pixel; accordingly, if $x(k)$ is located on the line, then it may be identified as impulse noise and removed. Other observations must be made to improve accuracy and prevent incorrect judgements, so the variable $v(k)$ is provided that is defined as follows (Lin and $\mathrm{Yu}, 2006)$.

\section{Definition 2.}

$$
v(k)=\frac{\left|x(k)-x_{c 1}(k)\right|+\left|x(k)-x_{c 2}(k)\right|}{2},
$$

where $\left|x(k)-x_{c 1}(k)\right| \leq\left|x(k)-x_{c 2}(k)\right| \leq\left|x(k)-x_{i}(k)\right|, 1 \leq i \leq 2 n+1, i \neq n+1, c 1, c 2$.

Note that $x_{c 1}(k)$ and $x_{c 2}(k)$ are selected as the pixel values that are closest to that of $x(k)$ of all adjacent pixels in the filter window $w\{k\}$. If the variable $v(k)$ is applied, then the line component in the filter window $w\{k\}$ will not be detected as noise but will be preserved because of the small $v(k)$.

The second problem is that when a pixel is a good pixel, if $u(k)$ is large, it is normally mis-detected to be an impulse noise. Such pixels might also exist on edges. Hence, it is important to distinguish noise components from edge components for effective noise filtering and edge preservation. To solve this problem, another variable $q(k)$ must be provided.

\section{Definition 3.}

$$
q(k)=\left|x(k)-\operatorname{med}\left(w_{3}\{k\}\right)\right|
$$

where $\operatorname{med}\left(w_{3}\{k\}\right)=\operatorname{med}\left(x_{1}(k), \ldots, x_{n}(k), 3 \diamond x_{n+1}(k), \ldots, x_{N}(k)\right)$. Therefore, if the variable $q(k)$ is applied, the edge components in the filter window $w\{k\}$ will not be identified as noise because the $q(k)$ value is small.

Lin and $\mathrm{Yu}(2004)$ has selected only above three variables $u(k), v(k)$ and $q(k)$ as the features. On the other hand, Liu et al. (2006) has selected four variables $u(k), v_{1}(k), v_{2}(k)$ and $q(k)$, where $v_{1}(k)=\left|x(k)-x_{c 1}(k)\right|$ and $v_{2}(k)=\left|x(k)-x_{c 2}(k)\right|$ are variables obtained by separating the variable $v(k)$ into two variables.

The third problem is that if the noise value is close to its neighbors such that the $u(k)$ value is not sufficiently large, as shown in Figure 3, the variable $u(k)$ will not be detected as noise. Of course, such noise pixels may not exist in line and edge components, so it is necessary to add another variable $r(k)$. 


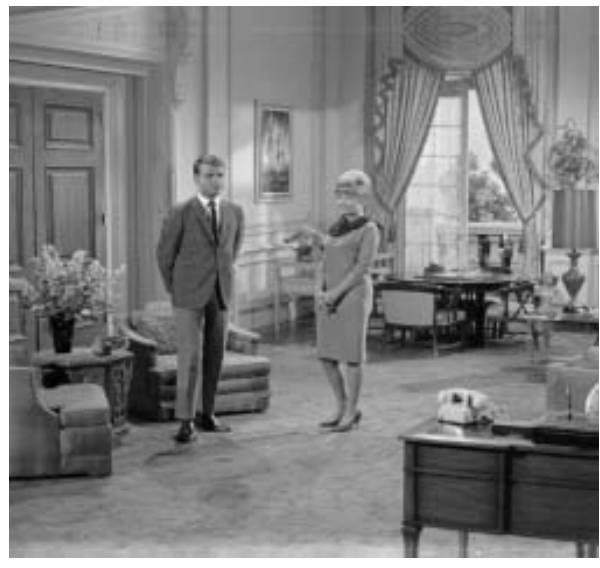

(a) Original image

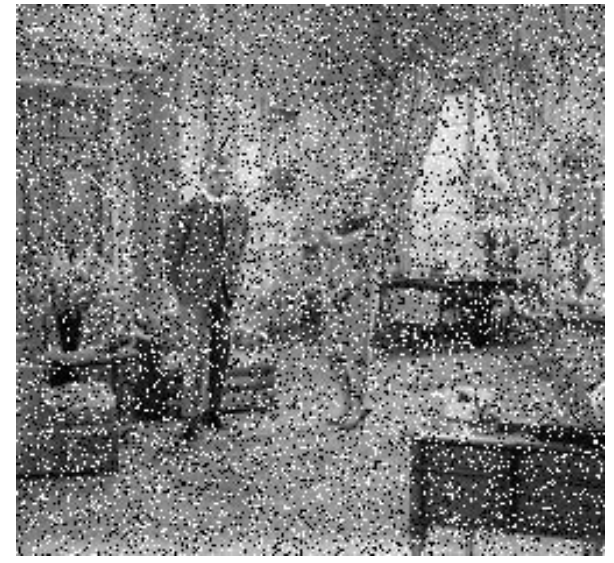

(b) Noisy image

Figure 4: Training Couple image

\section{Definition 4.}

$$
r(k)=|r(x(k))-r(\operatorname{med}(w\{k\}))|,
$$

where $r(x(k))$ and $r(\operatorname{med}(w\{k\}))$ are ranks of $x(k)$ and med $(w\{k\})$, respectively. The large value of $r(k)$ means that the rank order of the current pixel $x(k)$ is larger than the corresponding med $(w\{k\})$. It denotes that $x(k)$ is a noise pixel and must be filtered since the pixel close to the med $(w\{k\})$ has the less probability to be corrupted by impulse noise and the pixel close to one of two ends of the sorted samples is very possible impulse noise.

In this work, according to the above four variables $u(k), v(k), q(k)$ and $r(k)$ given in Equation (3.1) (3.4) the observation vectors are given by

$$
O(k)=(u(k), v(k), q(k), r(k)) .
$$

The observation vectors $O(k)$ are derived to extract the feature information from the filter window for training the SVM impulse detector. This feature information will also be used in the filtering stage.

\subsubsection{Training the $S V M$ impulse detector}

In order to obtain an optimal separating hyperplane for class distinction between noise-free and noisecorrupted classes, we extract the observation vector $O(k)$ in Equation (3.5) from a training image. We decided to use the $64 \times 64$ Couple image as shown in Figure 4, with $20 \%$ fixed-valued noise at random positions. This would also provide a set of supervised class labels for training the SVM. If we increase the noise ratio we obtain superior results over highly corrupted images; however, the number of support vectors increases. A higher size of the images produces a finer gray scale but increases the training and the execution time.

\subsection{Noise filtering with ASM filter}

\subsubsection{The ASM filter}

From the last section we got the binary flag map $F$ which elements give information about whether the pixel $x(k)$ is corrupted or not corrupted at location $k$ in noisy image $X$. If $x(k)$ has detected as a 
noise then it will go through ASM filtering process otherwise it will be kept unchanged. Here the size of filtering window is depend on the number of pixels which are noise free in current filtering window. Steps are given below for ASM filtering:

Step 1. Start with $3 \times 3$ filtering window $w\{k\}$ from $X$ and corresponding $3 \times 3$ window from binary flag map $F$.

Step 2. Find out how many pixels are detected as noise-free in current filtering window from corresponding binary flag window.

Step 3. If the number of uncorrupted pixels is less than half of the total number of pixels within the filtering window, we extend window size outward by one pixel in all the four sides of the window and go to Step 2.

Step 4. Replace the pixel detected as a noise by the median of the pixels that are classified as noisefree in filtering window.

One advantage of ASM filter is that, it makes sure that the details on the screen are not lost while the noise is suppressed.

\subsubsection{The improved noise filtering}

The mistakes that may be made by the SVM impulse detector are such that two problems may need to be solved. First, the undetected noisy pixels may remain in the restored image because the SVM impulse detector does not detect them to be noisy pixels. Second, the mis-detected pixels may appear in the restored image, causing the ASM filter to modify these pixels even though they are good pixels. Hence, a simple decision filter is required to improve the performance of the ASM-SVM filter.

The improved decision filter for detecting the corrupted pixels is based on the difference between the output $y^{\prime}(k)$ of the ASM filter in noisy image $X$ and the output value med( $\left.w^{\prime}\{k\}\right)$ of the ASM filter in adaptive filter window $w^{\prime}\{k\}$. Then, our decision filter employs the threshold $T$ to determine the final output $y(k)$,

$$
y(k)= \begin{cases}\operatorname{med}\left(w^{\prime}\{k\}\right), & \text { if }\left|y^{\prime}(k)-\operatorname{med}\left(w^{\prime}\{k\}\right)\right| \geq T, \\ y^{\prime}(k), & \text { otherwise. }\end{cases}
$$

Lin and Yu (2004) uses the decision filter based on the difference between the output of the firstpass filter and the output of the median filter in the filter window, with a filter similar to Equation (3.6).

A potential advantage of our adaptive switching filter is that when pixels not corrupted by impulse noise, they will not be affected the filtering, and when images are highly corrupted with impulse noise, the noise can be filtered by enlarging window size without destroying the details of images.

\section{Experimental Result Discussion}

In order to assess the performance of the proposed SVM-ASM filter, extensive computer simulation are carried out on 8-bits gray-scale images of $512 \times 512$ size. The images are Lena, Mandril, Bridge, Peppers, Cameraman and Airplane (Figure 5).

The two parameters while using RBF kernel for SVMs: kernel parameter $\gamma$ and penalty parameter $C$ are empirically taken to achieve the maximum filtering performance on the training Couple image. 


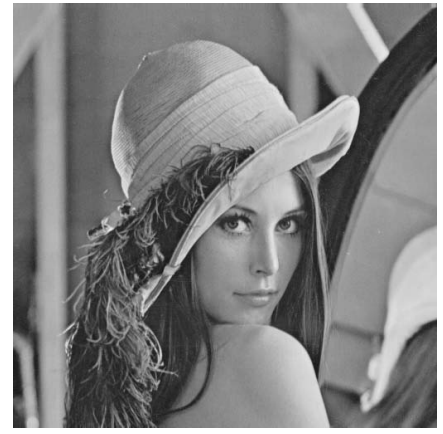

(a) Lena image

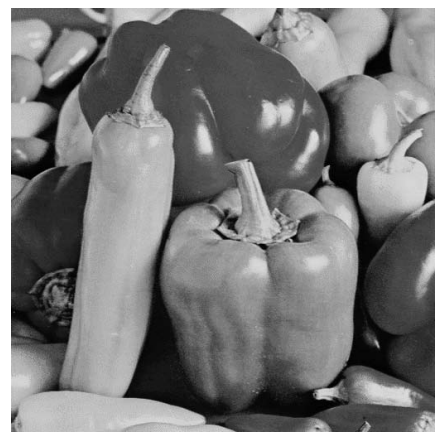

(d) Peppers image

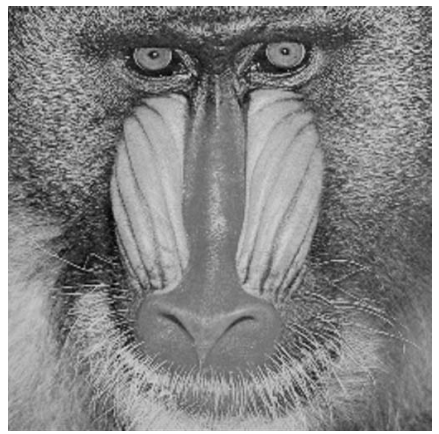

(b) Mandril image

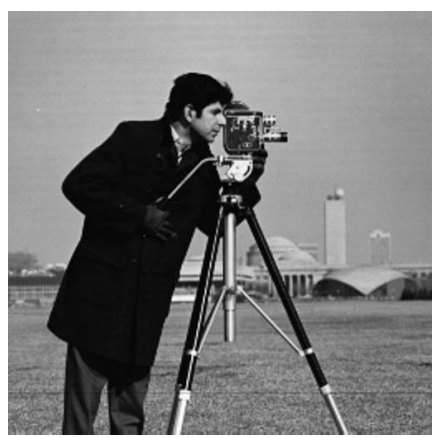

(e) Cameraman image

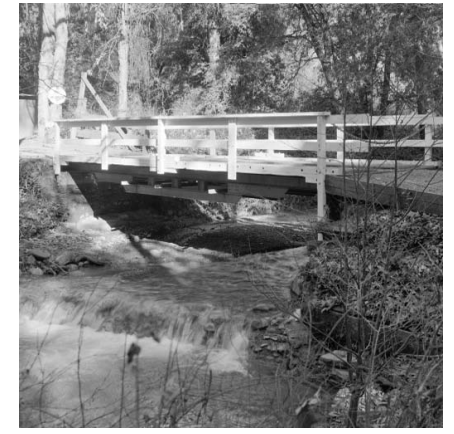

(c) Bridge image

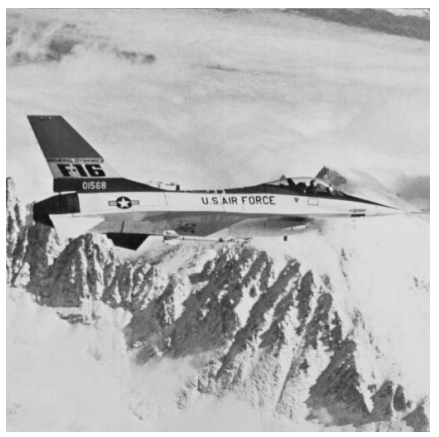

(f) Airplane image

Figure 5: Original test images

The performance of the proposed filter is evaluated in terms of the peak signal-to-noise ratio (PSNR) and the mean absolute error(MAE). The PSNR and MAE are given by

$$
\begin{aligned}
\text { PSNR } & =10 \log _{10}\left(\frac{\sum_{k} 255^{2}}{\sum_{k}(d(k)-y(k))^{2}}\right) d B, \\
\text { MAE } & =\frac{1}{H \times W} \sum_{k}|d(k)-y(k)|,
\end{aligned}
$$

where 255 is the peak gray-level of the image, $d(k)$ represents the value of the desired output, and $y(k)$ represents the value of the physical output. PSNR has been employed to measure the restoration performance quantitatively and MAE has also been taken as a quantitative measure to evaluate the preservation of edges and details.

In this work, for an impulse noise model with noise ratio $p$ can be described as follows:

$$
x(k)= \begin{cases}s(k), & \text { with probability } 1-p, \\ n(k), & \text { with probability } p,\end{cases}
$$

where $s(k)$ and $n(k)$ represent the original noise-free image pixel and the noise substitution for the original pixel, respectively (Chen and $\mathrm{Wu}, 2001$ ). There are two cases of noise distributions for impulse noise: fixed-valued impulse noise and random-valued impulse noise. For fixed-valued (also called salt-and-pepper) impulse noise in gray-level images with 8 bits per pixel, the values of the 


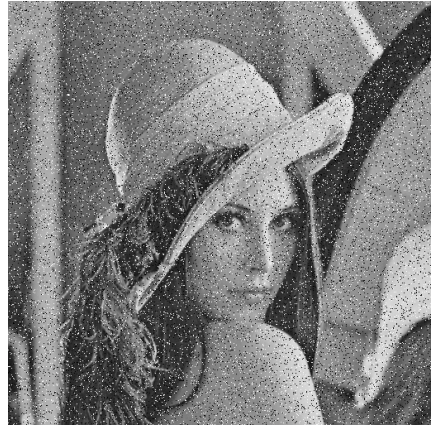

(a) Lena image with $10 \%$ fixed-valued impulse noise

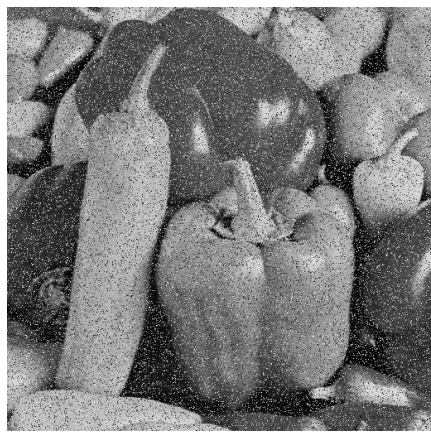

(d) Peppers image with $20 \%$ randomvalued impulse noise

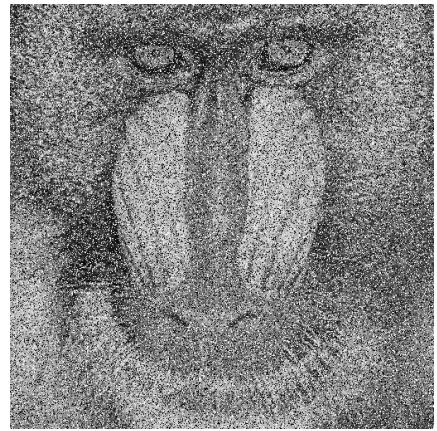

(b) Mandril image with $30 \%$ fixedvalued impulse noise

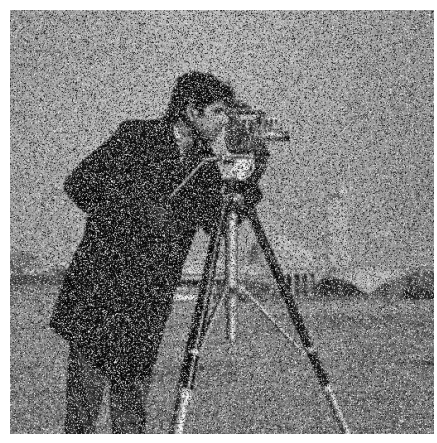

(e) Cameraman image with $40 \%$ random-valued impulse noise

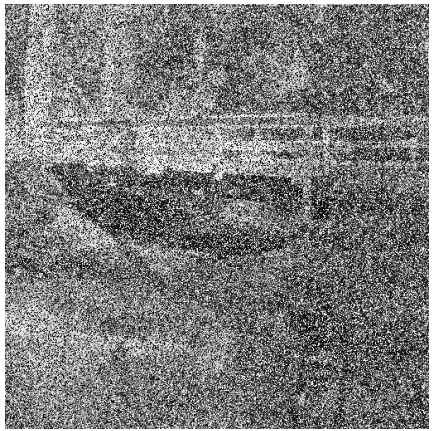

(c) Bridge image with $50 \%$ fixedvalued impulse noise

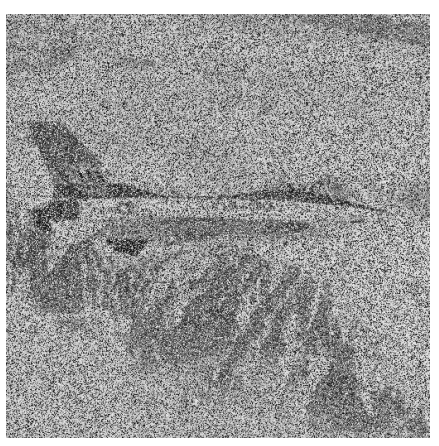

(f) Airplane image with $60 \%$ randomvalued impulse noise.

Figure 6: Noisy test images

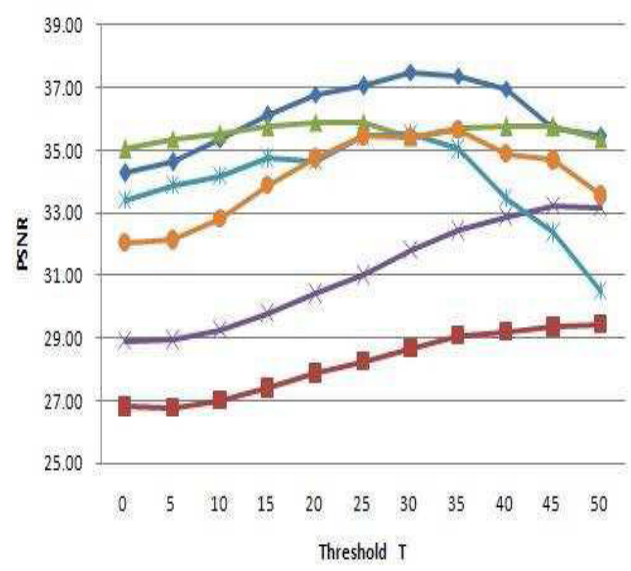

$\rightarrow$ Lena - - Bridge $\rightarrow$ Cameraman $*$ Mandril $*$ - Airplane -4 -Peppers

(a) Fixed-valued impulse noise

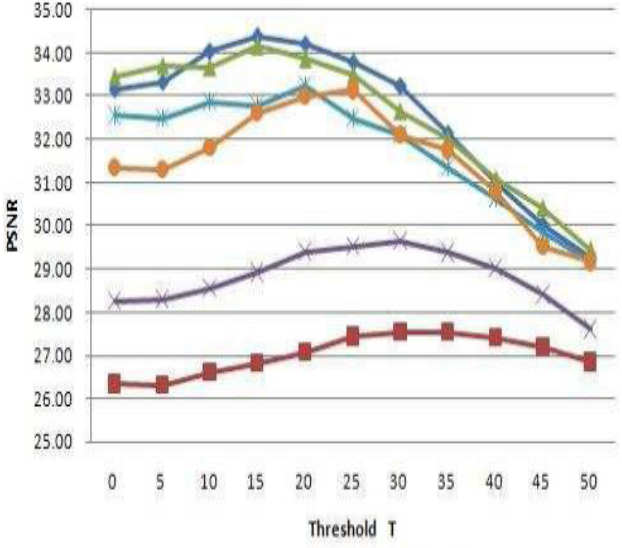

$\rightarrow$ Lena $\rightarrow$-Bridge $\rightarrow$-Cameraman + Mandril $\rightarrow$ - Airplane $-0-$ Peppers

(b) Random-valued impulse noise

Figure 7: Effect of threshold T with respect to PSNR for SVM-ASM filter 
Table 1: Comparative results in PSNR and MAE of filtering different images corrupted by fixed-valued impulse noise

\begin{tabular}{|c|c|c|c|c|c|c|c|c|c|c|c|c|}
\hline \multirow{2}{*}{ Filters } & \multicolumn{2}{|c|}{ Lena } & \multicolumn{2}{|c|}{ Bridge } & \multicolumn{2}{|c|}{ Cameraman } & \multicolumn{2}{|c|}{ Mandril } & \multicolumn{2}{|c|}{ Airplane } & \multicolumn{2}{|c|}{ Peppers } \\
\hline & PSNR & MAE & PSNR & MAE & PSNR & MAE & PSNR & MAE & PSNR & MAE & PSNR & MAE \\
\hline \multicolumn{13}{|c|}{$20 \%$ fixed-valued noise } \\
\hline MED & 30.98 & 3.55 & 24.99 & 8.68 & 30.51 & 2.73 & 25.75 & 7.87 & 29.66 & 3.42 & 29.87 & 4.12 \\
\hline CWM & 31.74 & 3.01 & 26.11 & 7.20 & 31.59 & 2.25 & 27.40 & 6.05 & 30.99 & 2.76 & 30.81 & 3.54 \\
\hline SWM-I & 34.40 & 1.09 & 27.22 & 3.62 & 32.43 & 1.24 & 29.74 & 2.53 & 32.55 & 1.19 & 33.29 & 1.35 \\
\hline SWM-II & 34.01 & 1.15 & 27.91 & 3.22 & 32.84 & 1.23 & 30.12 & 2.36 & 32.56 & 1.20 & 32.77 & 1.42 \\
\hline SD-ROM & 35.02 & 1.03 & 27.71 & 3.43 & 33.88 & 0.94 & 30.60 & 2.29 & 33.57 & 1.07 & 33.32 & 1.45 \\
\hline NN-ASM & 36.81 & 0.87 & 32.23 & 1.94 & 34.95 & 0.91 & 32.07 & 1.98 & 34.70 & 1.02 & 38.15 & 0.76 \\
\hline SVM-NFR & 36.83 & 0.89 & 28.39 & 3.09 & 35.34 & 0.91 & 31.64 & 1.97 & 34.99 & 0.94 & 34.73 & 1.18 \\
\hline SVM-EPR & 30.33 & 1.58 & 26.90 & 3.31 & 30.67 & 1.38 & 28.67 & 2.47 & 26.49 & 3.16 & 29.76 & 1.90 \\
\hline SVM-ASM & 37.42 & 0.85 & 29.31 & 2.60 & 35.25 & 0.92 & 31.96 & 1.81 & 35.54 & 0.89 & 35.44 & 1.14 \\
\hline \multicolumn{13}{|c|}{$40 \%$ fixed-valued noise } \\
\hline MED & 26.29 & 5.46 & 22.62 & 11.18 & 25.39 & 4.60 & 23.44 & 10.39 & 25.30 & 5.25 & 25.76 & 5.80 \\
\hline CWM & 24.71 & 5.77 & 22.16 & 10.68 & 23.99 & 5.19 & 22.67 & 9.86 & 24.08 & 5.67 & 24.20 & 6.31 \\
\hline SWM-I & 27.90 & 2.87 & 23.77 & 7.01 & 26.10 & 3.33 & 25.24 & 5.73 & 26.47 & 3.20 & 27.41 & 3.32 \\
\hline SWM-II & 24.53 & 4.32 & 22.27 & 7.87 & 24.15 & 4.38 & 23.27 & 6.65 & 23.96 & 4.42 & 24.17 & 4.72 \\
\hline SD-ROM & 27.07 & 3.01 & 23.60 & 6.75 & 26.05 & 3.02 & 25.53 & 5.12 & 26.32 & 3.38 & 26.63 & 3.55 \\
\hline NN-ASM & 32.28 & 2.07 & 28.39 & 4.18 & 30.81 & 2.08 & 28.44 & 4.35 & 30.43 & 2.31 & 33.10 & 1.80 \\
\hline SVM-NFR & 24.81 & 3.71 & 23.31 & 6.79 & 25.84 & 3.13 & 25.51 & 4.77 & 24.51 & 4.49 & 24.86 & 4.18 \\
\hline SVM-EPR & 20.09 & 7.65 & 19.41 & 9.99 & 16.80 & 9.93 & 19.62 & 9.37 & 19.08 & 9.68 & 20.13 & 7.19 \\
\hline SVM-ASM & 29.62 & 2.28 & 24.53 & 5.95 & 30.65 & 2.22 & 26.61 & 4.45 & 27.16 & 3.19 & 27.72 & 3.13 \\
\hline \multicolumn{13}{|c|}{$60 \%$ fixed-valued noise } \\
\hline MED & 20.03 & 10.33 & 18.80 & 16.30 & 19.44 & 9.22 & 19.00 & 15.99 & 19.61 & 9.77 & 19.65 & 10.71 \\
\hline CWM & 17.27 & 16.23 & 16.44 & 21.16 & 17.07 & 15.32 & 16.72 & 20.73 & 16.64 & 16.31 & 17.05 & 16.43 \\
\hline SWM-I & 20.63 & 7.80 & 18.90 & 13.69 & 19.55 & 8.43 & 19.91 & 11.94 & 19.93 & 8.01 & 20.10 & 8.51 \\
\hline SWM-II & 16.95 & 15.55 & 15.93 & 20.45 & 16.49 & 15.82 & 16.43 & 19.08 & 16.23 & 16.47 & 16.69 & 16.12 \\
\hline SD-ROM & 19.23 & 9.82 & 17.94 & 14.99 & 19.01 & 9.29 & 18.96 & 12.53 & 18.89 & 10.97 & 19.01 & 10.24 \\
\hline NN-ASM & 27.95 & 4.08 & 25.32 & 7.27 & 26.54 & 3.93 & 25.55 & 7.30 & 26.76 & 4.30 & 28.54 & 3.64 \\
\hline SVM-NFR & 15.22 & 19.32 & 14.49 & 23.06 & 14.69 & 19.04 & 14.75 & 22.21 & 13.89 & 21.54 & 15.38 & 19.10 \\
\hline SVM-EPR & 13.32 & 27.85 & 13.03 & 30.16 & 12.92 & 27.86 & 13.39 & 28.98 & 12.85 & 29.27 & 13.08 & 28.11 \\
\hline SVM-ASM & 27.35 & 4.45 & 18.95 & 14.01 & 25.46 & 4.82 & 21.54 & 11.11 & 22.09 & 8.10 & 23.90 & 6.77 \\
\hline
\end{tabular}

corrupt pixels are equal to 0 or 255 with equal probability(i.e., $p / 2)$. For random-valued impulse noise, however, the corrupt pixel values are uniformly distributed within the range of [0,255].

Fixed-valued and random-valued impulses are artificially injected into these images at noise ratios ranging from $10 \%$ to $60 \%$ at increments of $10 \%$. Figure 6 shows some of the noisy images used in this experiment.

In the first experiment, we investigate the influence of the threshold $T$ employed by the SVMASM filter. Figure 7 shows the the graph of the PSNR values versus the threshold $T$ for different test images. For the majority of images we tested, the threshold $T$ employed by the proposed filter lies approximately in the range of $[25,45]$ for the fixed-valued impulse and $[15,30]$ for random-valued impulse at $p=20 \%$. We observe that these threshold ranges are quite consistent and common for the fixed-valued impulse and random-valued impulse, respectively.

The second experiment is to compare the SVM-ASM filter with the other conventional filters, namely MED filter, CWM filter, SD-ROM filter, SWM-I and SWM-II filter, SD-ROM filter, NNASM filter and two existing SVM-based filters, i.e. SVM-NFR filter and SVM-EPR filter.

Table 1 and Table 2 show comparative restoration results in PSNR and MAE for filtering different 
Table 2: Comparative results in PSNR and MAE of filtering different images corrupted by random-valued impulse noise

\begin{tabular}{|c|c|c|c|c|c|c|c|c|c|c|c|c|}
\hline \multirow{2}{*}{ Filters } & \multicolumn{2}{|c|}{ Lena } & \multicolumn{2}{|c|}{ Bridge } & \multicolumn{2}{|c|}{ "Cameraman } & \multicolumn{2}{|c|}{ Mandril } & \multicolumn{2}{|c|}{ Airplane } & \multicolumn{2}{|c|}{ Peppers } \\
\hline & PSNR & MAE & PSNR & MAE & PSNR & MAE & PSNR & MAE & PSNR & MAE & PSNR & MAE \\
\hline \multicolumn{13}{|c|}{$20 \%$ random-valued noise } \\
\hline MED & 30.85 & 3.70 & 24.88 & 9.03 & 30.18 & 2.97 & 25.74 & 8.03 & 30.03 & 3.51 & 29.64 & 4.34 \\
\hline CWM & 32.32 & 3.07 & 26.20 & 7.41 & 31.72 & 2.41 & 27.48 & 6.23 & 31.23 & 2.99 & 31.12 & 3.66 \\
\hline SWM-I & 32.35 & 1.69 & 26.95 & 3.96 & 31.15 & 1.64 & 28.88 & 3.00 & 31.11 & 1.82 & 31.69 & 1.82 \\
\hline SWM-II & 32.27 & 1.75 & 27.48 & 3.65 & 31.79 & 1.62 & 29.31 & 2.86 & 31.37 & 1.8 & 31.74 & 1.89 \\
\hline SD-ROM & 33.74 & 1.30 & 27.42 & 3.69 & 32.93 & 1.14 & 29.51 & 2.74 & 32.66 & 1.31 & 32.95 & 1.63 \\
\hline NN-ASM & 18.14 & 10.58 & 17.61 & 11.50 & 17.01 & 12.03 & 18.66 & 10.25 & 16.72 & 12.51 & 16.62 & 12.71 \\
\hline SVM-NFR & 33.23 & 1.53 & 27.65 & 3.54 & 33.16 & 1.34 & 29.73 & 2.66 & 32.31 & & 32.84 & 1.63 \\
\hline SVM-EF & 25.65 & 3.81 & 23.51 & 5.42 & 24.29 & 4.2 & 24.69 & 4.59 & 24.63 & & 24.48 & 4.14 \\
\hline SVM & 34.59 & 1.29 & 27.37 & 4.17 & 34.39 & 1.10 & 29.48 & 3.06 & 33.53 & 1. & 33.64 & 1.53 \\
\hline \multicolumn{13}{|c|}{$40 \%$ random-value } \\
\hline MED & 26.24 & 5.46 & 22.76 & 11.15 & 25.80 & 4.4 & 23.29 & 10.5 & 25.62 & 5. & 25.66 & 5.91 \\
\hline CWM & 27.06 & 5.61 & 23.10 & 11.10 & 25.58 & 5.5 & 24.58 & 9.28 & 25.55 & 6. & 25.99 & 6.58 \\
\hline SWM-I & 27.38 & 4.17 & 23.48 & 8.04 & 25.86 & 4. & 25.04 & 6.57 & 26.14 & $4 .($ & 26.59 & 4.59 \\
\hline & 26.50 & 4.81 & 23.25 & 8.22 & 24.96 & & 24.76 & 6. & 24.73 & & 25.36 & 5.53 \\
\hline SL & 27.84 & 3.6 & 23.83 & 7.39 & 26.69 & 3 & 25.26 & 6.11 & 26.84 & 3. & 27.21 & 4.14 \\
\hline NN-ASM & 13.21 & 29.30 & 12.80 & 30.47 & 12.25 & 32. & 13.79 & 27.58 & 12.12 & 32. & 12.03 & 32.96 \\
\hline SVM- & 27.82 & 3.87 & 23.82 & 7.35 & 25.82 & 4. & 25.53 & 6.00 & 26.08 & 4. & 26.64 & 4.41 \\
\hline FPP & 18.91 & 12.33 & 17.85 & 14.75 & 18.61 & 12. & 18.46 & 13.83 & 18.06 & 13 & 18.30 & 13.15 \\
\hline SVM & 28.76 & 3.38 & 23.83 & 7.35 & 27.64 & 3.34 & 25.82 & 6.06 & 26.17 & 4.04 & 27.03 & 4.35 \\
\hline \multicolumn{13}{|c|}{$60 \%$ random-value } \\
\hline MED & 19.96 & 10.45 & 18.63 & 16.48 & 19.43 & 9.33 & 19.16 & 15.74 & 9.79 & 9. & 19.83 & 10.63 \\
\hline CWM & 21.50 & 12.36 & 19.02 & 19.22 & 18.88 & 16. & 21.06 & 14.99 & 18.88 & 18.61 & 19.71 & 15.87 \\
\hline SWM-I & 22.27 & 9.70 & 19.59 & 15.90 & 19.47 & 13 & 21.54 & 12.26 & 20.51 & 12.77 & 20.58 & 12.33 \\
\hline SWM-II & 20.69 & 12.32 & 18.65 & 17.56 & 18.43 & 15. & 20.60 & 13.65 & 18.42 & 17.37 & 19.19 & 15.16 \\
\hline & 22.32 & & 19.69 & 14.82 & 20.28 & 11. & 21.25 & 12.11 & 20.66 & & 20.87 & 11.22 \\
\hline NN-ASM & 11.53 & 43.31 & 11.15 & 44.95 & 10.52 & 48. & 12.02 & 41.42 & 10.47 & 48.00 & 10.31 & 49.27 \\
\hline NER & 20.91 & 10.87 & 18.48 & 16.68 & 18.42 & 14 & 20.77 & 12.73 & 18.82 & 14.30 & 18.95 & 13.87 \\
\hline SVM-EPR & 15.27 & 25.15 & 14.41 & 28.64 & 13.78 & 30.04 & 15.30 & 25.78 & 13.25 & 32.39 & 14.08 & 29.25 \\
\hline SVM-ASM & 21.63 & 9.67 & 19.62 & 14.44 & 20.12 & 9.75 & 21.05 & 12.40 & 19.31 & 13.12 & 22.02 & 9.79 \\
\hline
\end{tabular}

images corrupted by fixed-valued noise and random-valued noise at noise ratios $p=20 \%, 40 \%$ and $60 \%$, respectively. As shown in Table 1, the SVM-ASM filter and the NN-ASM filter significantly outperform the other filters for various noise conditions in terms of PSNR and MAE. Comparison with the SVM-ASM filter and two existing SVM-based filters shows the difference of the performances of these filters significantly increases as the noise ratio is high. Table 2 reveals that the SVM-ASM filter has satisfactory results with the SD-ROM filter; however, the SD-ROM filter does not work well for removing fixed-valued impulse noise as shown in Table 1. The performance of the NN-ASM filter fell off sharply for random-valued impulse noise. The SVM-ASM filter achieves significantly high PSNR and low MAE for all images tested. The performance of the proposed is robust and insensitive to noise ratio.

In addition, (to the quantitative evaluation presented above) a qualitative evaluation is necessary since the visual assessment of the processed images is ultimately the best subjective measure of the effectiveness of any method. Due to the lack of space we omit here the restoration results for the other corrupted figures, except for the Bridge image and Cameraman image given in Figure 6(c) and 6(e), respectively. Figure 8 and Figure 9 show the restoration results for the Bridge image with 50\% fixed-valued noise and Cameraman image with $40 \%$ random-valued noise, respectively. It is seen 


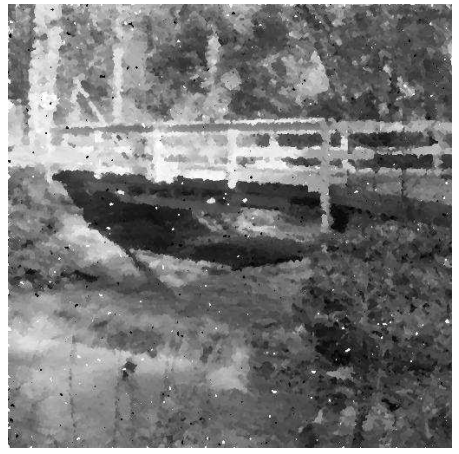

(a) MED

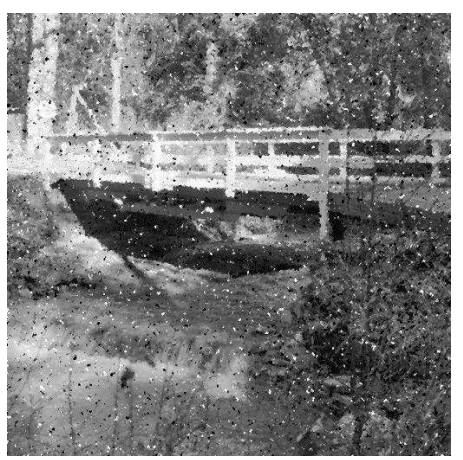

(d) SWM-II

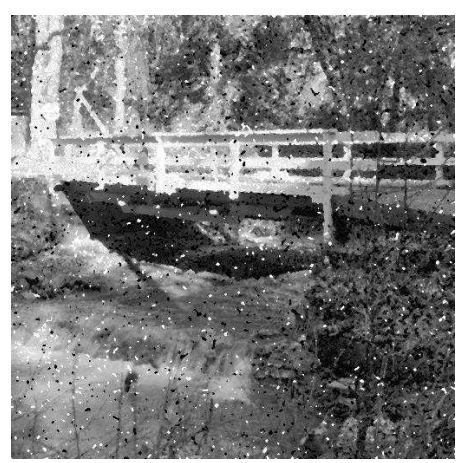

(g) SVM-NFR

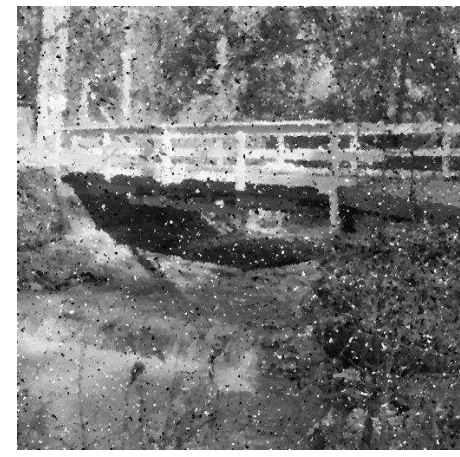

(b) CWM

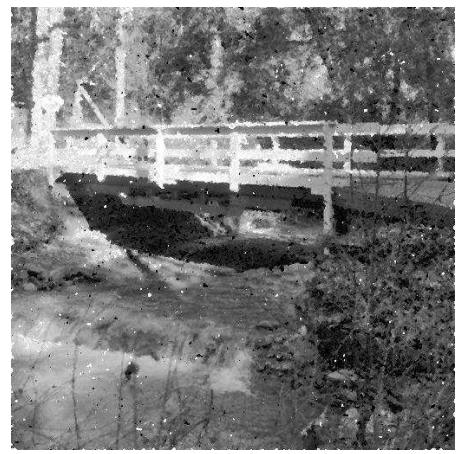

(e) SD-ROM

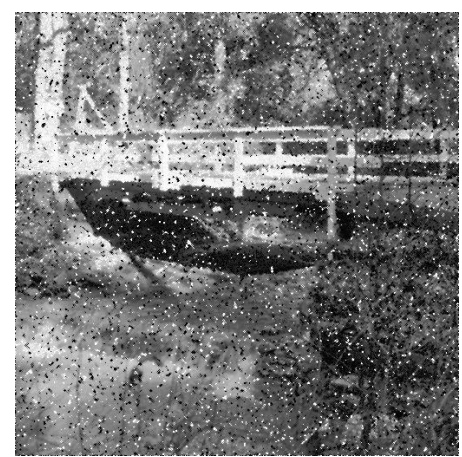

(h) SVM-EPR

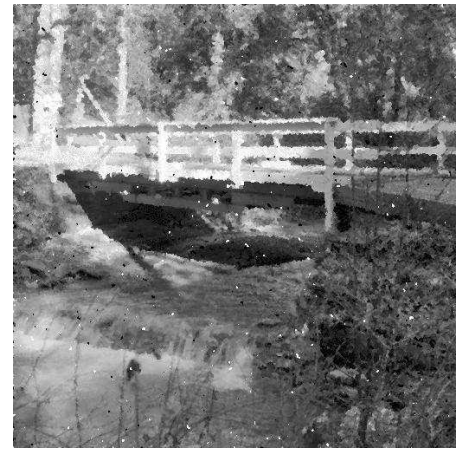

(c) SWM-I

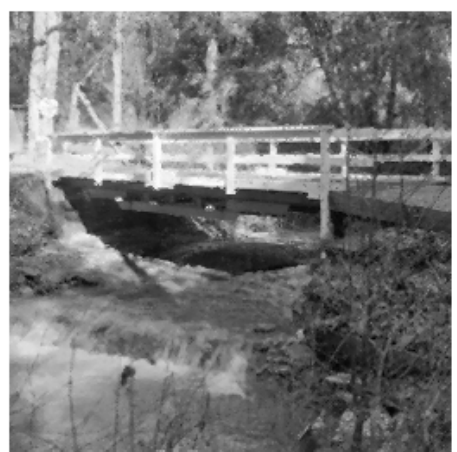

(f) NN-ASM

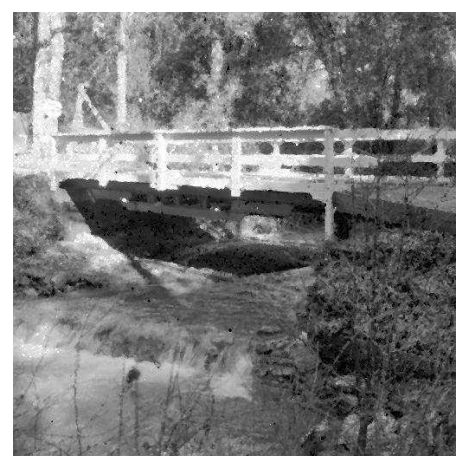

(i) SVM-ASM

Figure 8: Restoration results for Bridge image in Figure 6(c)

vividly from the Figure 8 that the SVM-ASM filter and the NN-ASM filter show superior results to the other filters mentioned in our paper. Figure 9 presents that the SVM-ASM filter seems like a similar results with the MED filter; however, if you look at Figure 9(a) in more detail, the MED filter produces the undesired outcomes that blur or distort image features. The restored image obtained by the NN-ASM filter contains considerable noise spots shown in Figure 9(f). Compared to the original images in Figure 5(c) and 5(e), it is clear that the SVM-ASM filter eliminated a fair amount of noise while preserving edge boundaries and fine details. 


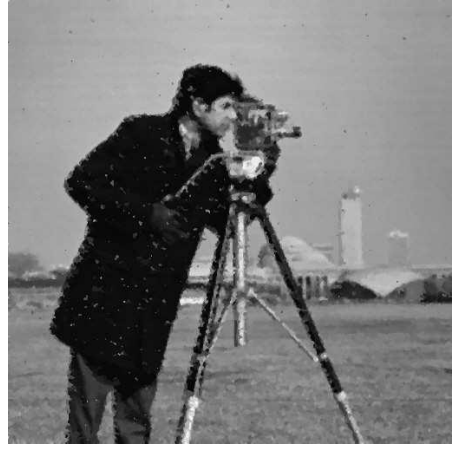

(a) MED

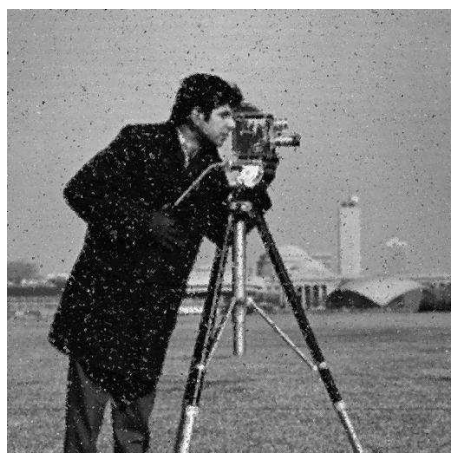

(d) SWM-II

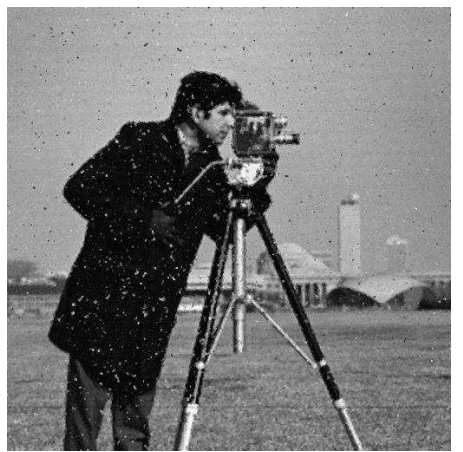

(g) SVM-NFR

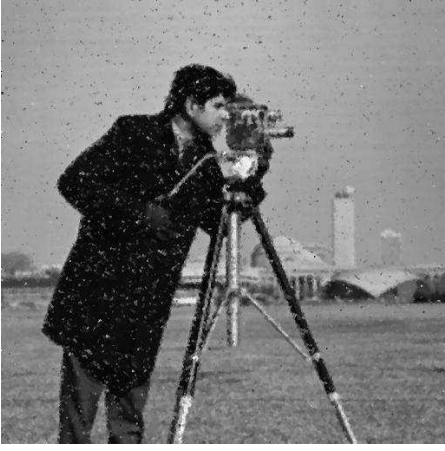

(b) CWM

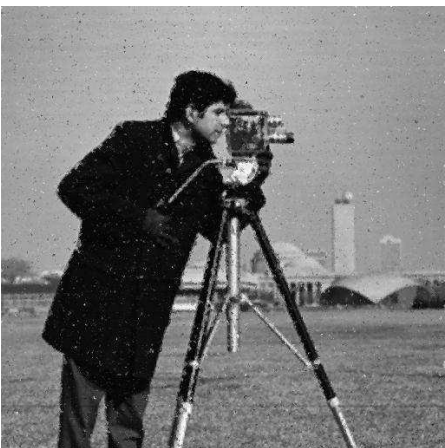

(e) SD-ROM

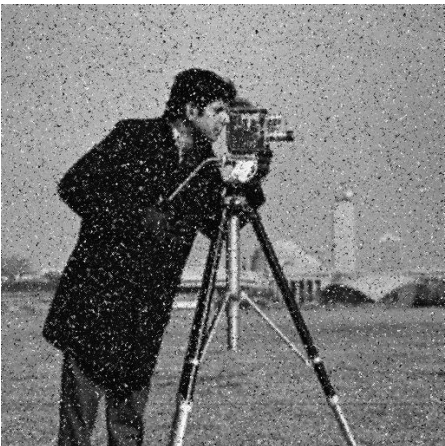

(h) SVM-EPR

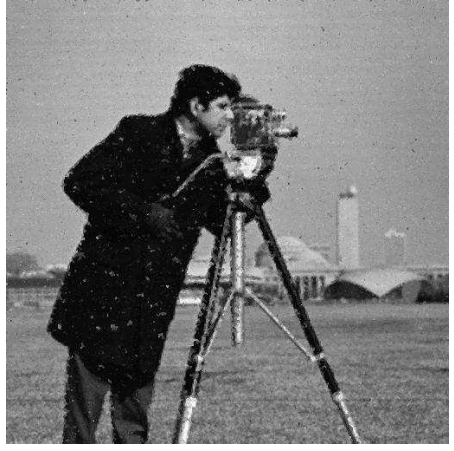

(c) SWM-I

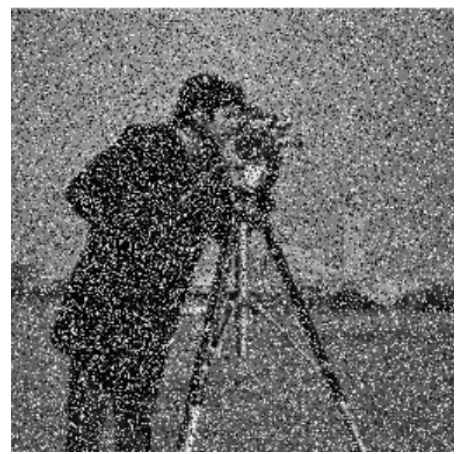

(f) NN-ASM

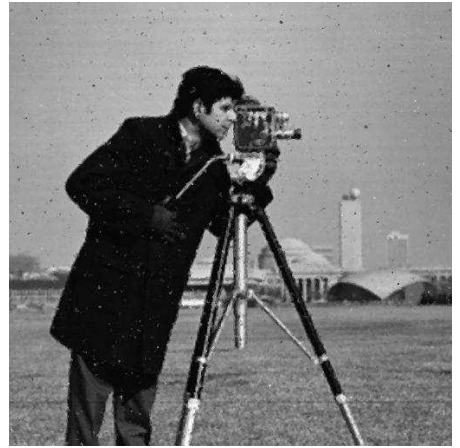

(i) SVM-ASM

Figure 9: Restoration results for Cameraman image in Figure 6(e)

\section{Conclusions}

This work presents a powerful two-stage filter called SVM-ASM filter, the ASM filter based on SVMs, for effectively reducing impulse noise in corrupt images while preserving image details and features. The proposed SVM-ASM filter is composed of two stages: efficient impulse detection based on SVMs and noise filtering with ASM filter. The SVM detector is modified by using the concept of rank order to improve the impulse detection capability. The former determines whether the pixels are corrupted by noise or not according to an optimal discrimination function. The latter implements the 
image filtering with variable window size on the noisy pixels to effectively remove the noisy pixels determined by the former. Due to its unbiased detection-filtering criteria, this filter treats both fixedvalued and random-valued noise even in highly corrupted images.

Extensive simulations indicate that the SVM-ASM filter performs significantly better in terms of noise suppression and detail preservation than a number of existing nonlinear filters with as much as $60 \%$ impulse noise corruption. The SVM-ASM filter is also shown to be extremely robust with respect to various test images and percentages of impulse noise.

\section{References}

Abreu, E. and Mitra, S. K. (1995). A signal-dependent rank ordered mean(SD-ROM) filter - a new approach for removal of impulses from highly corrupted images, International Conference on Acoustics, Speech and Signal Processing, 4, 2371-2374.

Apalkov, I. V., Zvonarev, P. S. and Khryashchev, V. V. (2005). Neural network adaptive switching median filter for image denoising, The International Conference on Computer as a Tool, 959962.

Chan, R. H., Ho, C. W. and Nikolova, M. (2005). Salt-and-pepper noise removal by median-type noise detectors and detail-preserving regularization, IEEE Transactions on Image Processing, 14, 1479-1485.

Chen, T. and Wu, H. R. (2001). Space variant median filters for the restoration of impulse noise corrupted images, IEEE Transactions on Circuits and Systems, 48, 784-789.

Cheng, H., Yu, Q., Tian, J. and Liu, J. (2005). Speckle reduction of SAR images using support vector machine in wavelet domain, Proceeding of SPIE 6043, 738-744.

Ganapathiraju, A., Hamaker, J. H. and Picone, J. (2004). Applications of support vector machines to speech recognition, IEEE Transactions on Signal Processing, 52, 2348-2355.

Gonzalez, R. C. and Woods, R. E. (1992). Digital Image Processing, Addison-Wesley publishing Co, New York.

Keerthi, S. S. and Lin, C. J. (2003). Asymptotic behaviors of support vector machines with Gaussian kernel, Neural Computation, 15, 1667-1689.

Ko, S. J. and Lee, Y. H. (1991). Center weighted median filters and their applications to image enhancement, IEEE Transactions on Circuits and Systems, 38, 984-993.

Lim, D. H. (2006). Robust edge detection in noisy images, Computation Statistics and Data Analysis, 50, 803-812.

Lim, D. H. and Jang, S. J. (2002). Comparison of two-sample tests for edge detection in noisy images, Journal of Royal Statistical Society D -The Statistician, 51, 21-30.

Lin, H. T. and Lin, C. J. (2003). A Study on Sigmoid Kernels for SVM and the Training of NonPSD Kernels by SMO-Type Methods, Technical report, Department of Computer Science and Information Engineering, National Taiwan University.

Lin, T. C. and Yu, P. T. (2004). Adaptive two-pass median filter based on support vector machines for image restoration, Neural Computation, 16, 192-206.

Lin, T. C. and Yu, P. T. (2006). Thresholding noise-free ordered median filter based on DempsterShafer theory for image restoration, IEEE Transactions on Circuits and Systems, 53, 1057-1064.

Liu, H., Sun, F. and Sun, Z. (2006). Image filtering using support vector machine, Lecture Notes in Computer Science, 3972, 533-538.

Moreno, H. G., Bascon, S. M., Ferreras, F. L. and Jimenez, P. G. (2003). Removal of impulse noise in images by means of the use of support vector machines, Lecture Notes in Computer Science, 2687, 559-566. 
Moreno, H. G., Bascon, S. M., Manso, M. U. and Martin, P. M. (2001). Elimination of impulsive noise in images by means of the use of support vector machines, XVI National Symposium of URSI, 1-2.

Sun, T. and Neuvo, Y. (1994). Detail-preserving median based filters in image processing, Pattern Recognition Letters, 15, 341-347.

Vapnik, V. (1998). The Nature of Statistical Learning Theory, Springer-Verlag, New York.

Zvonarev, P. S., Apalkov, I. V., Khryashchev, V. V. and Reznikova, I. V. (2005a). Neural network adaptive Switching median filter for the restoration of impulse noise corrupted images, Lecture Notes in Computer Sciences, 3656, 223-230.

Zvonarev, P. S., Apalkov, I. V., Khryashchev, V. V. and Reznikova, I. V. (2005b). Adaptive switching median filter with neural network impulse detection step, Lecture Notes in Computer Science, 3696, 537-542. 